\title{
Echos du groupe de travail EAE et nouvelles des tarifs de laboratoire
}

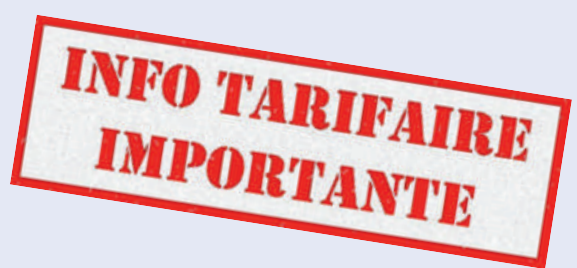

\section{Ernst Gählera , \\ Thomas Kessler ${ }^{b}$, \\ Susanne Christen ${ }^{c}$}

a Dr, vice-président de la FMH responsable du domaine Tarifs et conventions pour la médecine ambulatoire en Suisse

b FMH, Tarifs et conventions pour la médecine ambulatoire en Suisse

c Dr, FMH, Tarifs et conventions pour la médecine ambulatoire en Suisse
Correspondance:

FMH / Tarifs et conventions pour la médecine ambulatoire en Suisse

Froburgstrasse 15

CH-4600 Olten

Tél. 0313591230

Fax 0313591238

tarife.ambulant[at]fmh.ch

\section{Groupe de travail EAE - état actuel des négociations}

Lors de la séance du 27 novembre 2013 du groupe de travail EAE (efficacité, adéquation, économicité), les parties contractantes (FMH, santésuisse et curafutura) se sont accordées sur une convention commune concernant l'art. 56 al. 6 de la loi sur l'assurance-maladie (LAMal) lequel prévoit que «Les fournisseurs de prestations et les assureurs conviennent d'une méthode visant à contrôler le caractère économique des prestations.» Cette convention, qui sera soumise au Conseil fédéral pour information, a été élaborée par les parties contractantes dans le respect des dispositions légales. Elle détermine les quatre points-clés suivants:

1. L'analyse de la variance est la méthode statistique choisie pour l'examen de l'économicité des prestations médicales. Elle permet d'évaluer les écarts constatés entre les fournisseurs de prestations au sein de leur groupe de comparaison.

2. Le modèle d'analyse de la variance actuellement utilisé comprend des variables démographiques et explicatives. A l'avenir, le modèle sera perfectionné conjointement par les fournisseurs de prestations et les assureurs, et il sera complété par l'ajout de variables de morbidité.

3. Les variables explicatives seront testées et validées dans de nouveaux modèles statistiques en se basant sur un ensemble de données optimisé en permanence.

4. En plus du mandat attribué par le législateur de déterminer une méthode statistique, fournisseurs de prestations et assureurs ont convenu d'uniformiser et de professionnaliser le contrôle de l'économicité par la Commission paritaire de confiance (CPC). La réglementation requise fera l'objet d'une convention commune.

Dans le cadre du groupe de travail EAE, trois sousgroupes traitant chacun de sujets spécifiques s'attellent actuellement à la recherche de solutions appropriées:

- Le sous-groupe 1 «ANOVA» planche sur l'amélioration des méthodes statistiques (méthode des coûts moyens et analyse de la variance) afin d'obtenir moins de fournisseurs de prestations faux positifs ou faux négatifs lors du contrôle de l'économicité. Il s'agit de déterminer les variables spécifiques à chaque groupe de spécialiste pour l'analyse de la variance permettant de déclarer de manière empirique que les coûts de la prestation fournie par le médecin sont valides dans son collectif de comparaison. Les travaux sont en cours.

- Le sous-groupe 2 «CPC» traite des procédés et des structures des commissions paritaires cantonales et régionales qui doivent être uniformisées et professionnalisées. A l'avenir, il n'y aura probablement plus que trois commissions paritaires de confiance régionales (Suisse alémanique, Suisse romande et Tessin). Le règlement des CPC est actuellement en consultation auprès des parties contractantes.

- Le sous-groupe 3 «Homogénéisation des collectifs de comparaison» poursuit le même objectif que le sous-groupe 1 «ANOVA» en affinant les collectifs de comparaison grâce à de meilleures données de base et en tenant compte des facteurs de morbidité. Les travaux en cours visant à affiner les collectifs de comparaison se déroulent en collaboration avec les sociétés de discipline médicale et prévoient un système dit évolutif.

De plus, les parties contractantes sont en train d'élaborer une convention-cadre qui reflète dans le détail les modalités du contrôle de l'économicité et qui définit sous la forme d'une feuille de route les travaux à accomplir et leur échéance. La prochaine séance du groupe de travail EAE aura lieu le 22 janvier 2014.

\section{Laboratoire - les décisions relatives au tarif Point-of-Care}

En lien avec la prise de position de la FMH basée sur des faits, le conseiller fédéral Alain Berset a décidé d'intégrer 33 «analyses rapides» dans le tarif Pointof-Care (POCT). De plus, au vu des résultats du monitorage, il a choisi de compenser la trop forte baisse des laboratoires au cabinet en allouant 35 millions de francs aux analyses. La FMH se réjouit que son modèle de coûts ait été sélectionné pour la revalorisation des 33 prestations dans le tarif POCT. Mais la prise en compte et la valeur des coûts indirects sont des questions encore en suspens, de même que les autorisations de facturer. Les décisions suivantes ont d'ores et déjà été prises:

- Les pharmaciens ne pourront pas facturer les «analyses rapides» selon le nouveau tarif.

- Conformément à l'art. 54 al. 1 let. a OAMal, tous les laboratoires de cabinet peuvent facturer les positions du chapitre «analyses rapides», soit aussi bien les médecins de premier recours 
(médecine interne générale, pédiatrie, médecins praticiens au sens de l'annexe 1 de l'ordonnance sur les professions médicales) que les médecins spécialistes.

La question de savoir quand la structure tarifaire POCT entrera en vigueur reste ouverte.

\section{La trop forte baisse des laboratoires au cabinet} sera compensée grâce à 35 millions de francs

\section{supplémentaires alloués aux analyses.}

\section{Laboratoire - valeur transitoire du point tarifaire}

Etant donné que la structure tarifaire POCT ne pourra pas entrer en vigueur au $1^{\text {er }}$ janvier 2014, le supplément de transition - actuellement 1,1 point tarifaire - sera relevé à $1,9 P T=C H F 1.90$ à partir du $1^{\text {er }}$ janvier 2014 jusqu'à l'entrée en vigueur du tarif POCT afin que les 35 millions soient à la disposition des médecins dès le mois de janvier.

\section{Laboratoire - QUALAB: nouvelles valeurs de l'intervalle de tolérance à partir du 1 er janvier 2014}

La commission QUALAB a procédé au réajustement de plusieurs intervalles de tolérance dans les contrôles de qualité internes (CQI) et externes (CQE). Cela concerne notamment les paramètres suivants: gaz du sang (pH et p CO2), lactate déshydrogénase (LDH), acide urique, 25-hydroxycholécalciférol ou vitamine D3. Les nouvelles valeurs de l'intervalle de tolérance sont applicables à partir du

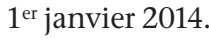

Vous trouverez les directives et les listes relatives aux contrôles de qualité internes et aux contrôles obligatoires de qualité externes pour 2014 sur le site www.qualab.ch.

\section{Prendre le pouls de l'actualité. L'offre groupée de la FMH.}

Bulletin des médecins suisses, Today's Press, FMH-Flash. Gratuit pour nos membres. 